\title{
智能电网时代电力信息通信技术的应用
}

\author{
赵鹤白杨刘阳
}

国网河南省电力公司周口供电公司

DOI:10.32629/hwr.v3i12.2606

[摘 要] 现在随着社会的进步,科技的发展,互联网时代已经来临。互联网已经运用到了各个领域,电力通讯技术也不例外。然而电力信息通信 技术在智能电网建设过程中起着十分重要的作用。电力通信技术给信息的传输和融合提供了方便,给我国的电力事业奠定着一定的基础。为了 使网络使用环境更加安全高效,电力信息通信技术在智能电网时代里的应用,就成了当今时代不得不进行深入研究探讨的问题。

[关键词] 智能电网; 电力信息; 通信技术; 应用

\section{1 智能电网与电力信息通信技术的定义}

将智能化、信息化和电力技术融合起来, 实现电网的自动化管理, 这就 是今天我们见到的智能电网, 它可以充分地发挥出电力系统的性能, 对电 力利用效率的提高以及公司经济效益的提升有着非常重要的作用。同时, 安全性作为智能电网的核心要求, 是其最基本的保证, 是整个电网正常运 行的保证, 也是对比传统电力系统的突出优势所在。

电力从生产、传输、调配和使用等环节, 其环节众多步骤繁杂, 需要采 取高效的调度和调配通信手段才可以保证电网系统的正常运转, 顺利实现 其经济效益与安全目标。电力信息通信技术便是为此而生, 它贯穿于整个 电力系统的大部分环节, 在其中起到一个集中调度和协调管理的作用, 大 幅度提高整个电网系统的运转效率和安全性, 二者的相互依托相互支持, 使得其存在十分紧密的联系。

\section{2 电力信息通信技术在建设智能电网中所起到的作用}

2. 1 电力信息通信技术在智能光纤通信网络建设中, 是最基础的也是 最关键的。目前, 社会在电力系统的需求上提出了高标准, 系统通信的数据 量逐渐增加, 相对传统的光纤通信技术已经无法适应系统的运行。为此, 要想让效率有所提高, 就需要在网络智能化技术上进行广泛地应用, 构建 更高效、更安全的智能光纤通信系统。

2. 2 进行电力通信接入网建设时, 一般就是智能电网必须和用户端进 行连接, 将电力资源给予用户, 意思就是要将智能电网系统和用户用电之 间形成通信, 为此, 就必须要用通信系统进行辅助。

\section{3 智能电网时代对电力通信技术提出的要求}

3. 1 电力通信平台的多样化。智能电网的构建需要保障电力通信平台 的多样化。近年来, 电力行业的发展主要是将电力通信平台与智能技术进 行有效地结合, 从而满足电力企业的实际需求。由于我国的用电客户较多, 因此用电需求也相应较多, 电力企业需要统一规划电力通信平台。电力企 业需要按照规定的内容来对电力通信平台进行开放, 除了要满足用户需求 之外, 还需要跟上时代进步, 及时维护、更新。

3. 2 电力通信平台的保密性。智能电网当中包含大量的数据信息, 所以 引入电力通信系统来提升智能电网的保密性。目前的电力通信系统仍然不 够完善, 会被外界因素影响, 出现信息流失现象, 为了避免产生这一现象, 需要提前做好防护措施, 提高电力通信平台的保密性。

\section{4 电力信息通信技术}

4. 1 光纤电力通信技术。电力信息通信技术中光纤电力通信技术是非 常重要的组成部分, 电力信息通信技术当中发挥着非常重要的作用。光 纤电力通信技术将光导纤维作为介质, 用来对不同信号的信息技术进行 传输。这种技术优势就是, 非常安全而且承载量非常大。使用绝缘材料 制作光纤电力通信技术, 同时经常利用多芯来组成光缆, 有助于快速的
进行传输, 信息占用的空间比较小, 传输质量有所保证, 应用光纤通信技 术更加广泛。

4.2 通信网络技术。我国经济的不断进步发展, 电力工程技术水平也在 不断的提高, 从质量与数量上有所改变, 同时不断应用微波电路, 信息系统 更加自动智能化。为了推动电力工作的不断进步发展, 管理水平的逐渐提 高, 加强通信网络的动态监控工作, 保证电力系统顺利运转。

4. 3 智能电力设备技术。智能电力设备主要是电子技术和储能技术两方 面构成, 新能源使用可能会给输电系统带来影响, 这种现象出现时, 电子技 术这个现代技术可以被应用, 这个技术首先处理电能之后运输给用户, 从而 确保电能供应稳定。

\section{5 智能电网时代对电力信息通信技术的具体应用}

5. 1 在新能源中的应用。近年来我国的能源贾缺问题变得越来越严重, 并使得能源成为制约我国社会发展的一个重要因素。因此在进行智能电网 的建设过程中, 也就需要积极应用到多种新能源技术, 并进行能源的最优化 分配, 只有这样才能够为我国社会的进一步发展提供一个良好的基础。通过 将电力通信信息技术应用到智能电网技术中, 能够根据该电力系统的实际 运行情况来进行接口的合理设置, 并进行新能源的介入处理。此外通过电 力信息通信系统的合理应用, 也能够满足新能源发电的实际需求, 并进行 各种能源的有效控制。可以说在智能电网时代中通过电力信息通信技术的 合理应用, 能够有效满足新能源领域的各种需求, 并使得我国电能的使用 效率以及使用合理性得到有效的提升。

5. 2 电力信息通信技术在配电领域的具体应用。目前配电网络的运行 主要依赖于信息通信技术, 要想让配电领域更好的运行, 必须更加注重信 息通信技术的应用。经济型是配电网建设的考虑因素, 同时稳定性和可靠 安全性也是其重要的考虑因素。为了让配电网络更加稳定、安全必须有效 的引进先进的信息通信技术。通过先进的信息通信技术支持我国配电网向 着更加智能、安全、快速的方向进一步发展, 也有效地促进智能电网的快 速建设。

5.3 在输电领域中的应用。为了满足人们的用电需求, 也就要求输电系 统能够实现远距离、低耗损以及大容量的输电要求, 并在此过程中尽可能 地使用清洁能源, 只有这样才能够确保输电系统能够满足人们的实际用电 需求。在输电领域中通过电力通信信息技术的应用, 能够对输电线路的状 态以及输送能力进行实时的监控, 并借此来提升整个输电系统的输电效 能。此外通过电力信息通信技术的应用, 也能够完成各种输电信息以及输 电设备运行信息的采集与分析工作, 这样就能够对输电系统的运行状态有 一个清晰的掌握, 并能够在出现了输电故障的情况下迅速对该故障进行解 决, 从而确保整个输电系统的运行稳定性以及安全性。

5.4 在变电领域中的应用。智能变电站, 是当今电力系统不可或缺的关 


\title{
某电厂调速器事故配压闵误动作原因分析与处理
}

\author{
李志清 \\ 新疆伊犁河流域开发建设管理局 \\ DOI:10.32629/hwr.v3i12.2574
}

[摘 要] 某水电站的发电机组调速器利用事故停机系统, 为主配压阀控制系统在发生故障提供紧急停机的功能。然而在调速系统在调试的过程 中, 其事故配压㑈误动作, 根据其事故的原由作出分析, 将电气和液压系统作为切入点,最终找到误动作的原因, 因为调速器的油管内压不稳定以 及位移传感器反馈不到位,引起事故配压伡的油管内压下降。最终对事故配压伡误动原因提出了一些的处理方案,确保水电站的稳定运行。

[关键词]电厂; 调速器; 事故配压㑈; 误动作

\section{前言}

某市一水电站的水轮发电机组调速器出现事故配压阀误动作现象, 严 重影响到水电站的正常运行, 技术人员采取紧急行动, 分析事故原因。对调 速器液压系统管路进行进行优化, 并对位移传感器进行了更换, 经过验证, 其运行状态良好。

\section{1 某电厂工程背景}

该水电站以水力发电为主。水电站的电机组总容量为 $320 \mathrm{KW}$, 电机组 包括了四组水轮发电机组, 每一台的容量为 $80 \mathrm{MW}$ 。PID微机控制为该水电 站使用的电液调速器。在工作状态下, 额定油压强为 4.0 兆帕。为了保证 安全性, 水电站电机组采用的关闭方式为两段关闭方式, 该水电站同时 安装了事故配压阀以及分段关闭阀, 事故配压阀采用杭州三和电控设备 有限公司生产的产品。在水电厂技术人员对 2 号机组调试时, 甩 $100 \%$ 负荷 后准备重新增至额定负荷进行调速器事故低油压试验, 当机组增到满负 荷时, 事故配压阀出现误动, 导致机组导叶迅速关闭。对此从事故配压阀 电气和液压两, 方面进行试验分析, 经过仔细分析研究, 最终找到了简单 可行的解决方案。

\section{2 某水电站事故配压阀的工作原理}

在大中型水电站, 经常使用事故配压阀来确保水轮发电机组在快速运 行过程中的稳定状态 ${ }^{[1]}$ 。水轮发电机组的停机原理是当机组的转速太快, 其主配压阀的活塞将闭合停止动作, 那么会导致调速器的关闭导水机构作 用失效, 接力器和主配压阀的油路随之断开, 接力器停止运作, 水轮发电机 组停机。该水电站使用的事故配压阀为集成产品, 还包括了油阀以及电磁 阀。在水轮发电机组的正常运行状态下, 先导电磁阀让连接油管与其中某 一油箱接通, 事故配压阀并不参与到系统的控制工作中, 它将作为主配压 阀和导叶接力器的一条通路 ${ }^{[2]}$ 。当在紧急事故停机的情形下, 事故配压阀
将起到控制作用。

(1) 当调速器的主配压阀出现拒动现象时, 其关闭导水机构作用失效, 水轮发电机组的转速奛升, 过速装置在感应机组处于非正常运行状态时, 先导电磁阀接收到速装置的保护信号, 出现换向动作, 切断油路, 油路回流, 事故配压阀受到油压差的影响, 其阀芯移动换向, 油箱中的压力油流入接 力器, 迅速将水轮发电机组停机 ${ }^{[3]}$ 。(2) 机械液压的过速装置开始运作, 保 护装置生效, 回油油路与接口连通, 故配压阀受到油压差的影响, 其阀芯移 动换向, 油箱中的压力油流入接力器, 迅速将水轮发电机组停机。

\section{3 调速系统液压原理}

在水轮发电机组正常运行状态下, 具备足够压力的流量信号将会通过 引导阀, 进而到达主配压阀, 这种流量信号是由PID调节器所释放的 ${ }^{[4]}$ 。流 量信号会被主配压阀二次放大, 并利用接力器达到对导叶接力的控制作用, 通过改变导叶的开度来降低或者增水轮发电机组负荷大小 ${ }^{[5]}$ 。

\section{4 某水电站事故配压阀误动作原因}

在2019年6月, 工作人员对该水电站的三号水轮发电机组进行调试工 作时, 在达到一定负荷状态下, 将三号水轮发电机增加到额定负荷, 进行事 故低油压试验, 证明其工作系统是否良好。然而在三号水轮发电机组增加 到满负荷状态的过程中, 事故配压阀出现误动作现象, 三号水轮发电机组 导叶迅速关闭。在机组开机以及停机的过程中, 事故配压阀的误动作只发 生在紧急停机状态下。且工作人员对机组的相关部位和构件进行检查, 没 有排查出故障位置。再调出机组运行系统的实时监控记录中, 工作人员发 现当三号水轮发电机组快上升到满负荷时, 其导叶位置已经超出了百分之 七十八拐点, 随之相关的装置动作, 输出复归分段关闭阀的信号, 事故配 压阀接收信号并动作, 但工作人员看到监控并没有输出事故配压阀的动作 信号。

阻碍, 而电力信息通信技术的应用, 能够有效地保障智能电网的高效便捷 性和安全可靠性, 推动了智能电网时代的不断发展和进步, 保障智能电网 的安全性, 给人们的生活带来了很多便携之处。因此, 我们应当对电力信息 通信技术在智能电网中的应用加以重视, 并根据智能电网的发展, 对电力 信息通信技术进行不断地改进和完善。

\section{[参考文献]}

[1]卢彦飞.试论智能电网时代电力信息通信技术的应用和研究 [J].电 子世界,2017(01):118-119.

[2]李宁宁.试论智能电网时代电力信息通信技术的应用和研究 [J].城 市建设理论研究(电子版),2017(12):17-18.

[3]刘洋,李新,滕子贻. 智能电网时代电力信息通信技术的应用探讨 [J]. 现代国企研究,2017(16):147. 泛, 没有电力信息通信技术的支撑和维护, 智能电网时代的发展就会受到 